\title{
Effects of lower limb amputation on the mental rotation of feet
}

\author{
Carolin Curtze $\cdot$ Bert Otten $\cdot$ Klaas Postema
}

Received: 25 April 2009 / Accepted: 19 October 2009 / Published online: 10 November 2009

(C) The Author(s) 2009. This article is published with open access at Springerlink.com

\begin{abstract}
What happens to the mental representation of our body when the actual anatomy of our body changes? We asked 18 able-bodied controls, 18 patients with a lower limb amputation and a patient with rotationplasty to perform a laterality judgment task. They were shown illustrations of feet in different orientations which they had to classify as left or right limb. This laterality recognition task, originally introduced by Parsons in Cognit Psychol 19:178241, (1987), is known to elicit implicit mental rotation of the subject's own body part. However, it can also be solved by mental transformation of the visual stimuli. Despite the anatomical changes in the body periphery of the amputees and of the rotationplasty patient, no differences in their ability to identify illustrations of their affected versus contralateral limb were found, while the group of able-bodied controls showed clear laterality effects. These findings are discussed in the context of various strategies for mental rotation versus the maintenance of an intact prototypical body structural description.
\end{abstract}

\section{Curtze $(\bowtie) \cdot$ K. Postema}

Department of Rehabilitation Medicine,

Center for Rehabilitation, University Medical Center Groningen,

University of Groningen, P.O. Box 30.001,

9700 RB Groningen, The Netherlands

e-mail: c.curtze@ rev.umcg.nl

C. Curtze $\cdot$ K. Postema

Graduate School for Health Research,

University of Groningen, Groningen, The Netherlands

B. Otten

Center for Human Movement Sciences,

University of Groningen, Groningen, The Netherlands

B. Otten $\cdot$ K. Postema

School of Behavioral and Cognitive Neurosciences,

University of Groningen, Groningen, The Netherlands
Keywords Mental rotation - Motor imagery · Body representation $\cdot$ Amputation $\cdot$ Rotationplasty

\section{Introduction}

Our brain maintains multiple representations of the human body. One of them is the "body structural description", a topological map of the human body in general (CorradiDell'Acqua et al. 2009; Schwoebel and Coslett 2005; Buxbaum and Coslett 2001; Sirigu et al. 1991; Pick 1922). In addition, for the spatial organization of movements, our brain maintains a dynamic mental representation of our own body and its relation to the external world. This so-called "body schema" (Head and Holmes 1911) is an on-line, real-time mental representation of one's own body.

Neuropsychological studies suggest that the mental simulation of movement is guided by our body schema just like real motor actions (de Lange et al. 2005; Parsons 1994; Schwoebel et al. 2001). During the mental simulation of movements, the motor system is subliminally activated; only the actual execution of the motor act is suppressed. This parallelism between imagined and actual movements is evidenced by functional neuroimaging, showing large overlaps in brain activity between the two. The superior parietal cortex, the intraparietal sulcus, and the adjacent rostral-most part of the inferior parietal lobe were found to be highly active during mental rotation of body parts (Bonda et al. 1995; Parsons et al. 1995), just as during real movements. Further, chronometric findings revealed that mental rotation is subject to the same anatomical constraints as actual motor behaviour (e.g. Parsons 1994).

In the present study we examined how physical changes in lower limb amputees affect these phenomena. After amputation of a limb, no sensory input will reach the 
deafferented regions of the cortex. Peripheral input is vital for the formation and stability of the cortical sensorimotor map. Through movement, the cortical representations of limbs are expanded (e.g. Gaser and Schlaug 2003), and consequently the loss of a limb results in shrinkage of these representations (e.g. Knecht et al. 1996). In spite of this cortical reorganisation, many amputee patients experience a phantom limb. Some amputees describe their phantom as paralysed, while others can move it voluntarily (Melzack 1992; Ramachandran and Rogers-Ramachandran 2000). Blakemore et al. (2002) suggest that the motor control system can counteract the discrepancies between predicted and actual consequences of the motor command by modifying the forward model towards a no-movement prediction.

To quantify the effect of lower limb amputation on the mental rotation of feet, we used the laterality recognition task, first introduced by Parsons (1987). In this task, illustrations of hands or feet are presented in different views and orientations in random sequence. In the present study, participants are asked to classify foot illustrations according to their laterality as rapidly and accurately as possible by pressing a key. Participants were instructed to place their feet under the table (out of sight) in a forward pointing position and not to move them throughout the experiment. This task has been shown to elicit implicit mental rotation of one's own physical counterpart, until it is aligned with the position of the stimulus illustration. The more the orientation of the stimulus matches the orientation of the biological counterpart, the faster and more accurately it is recognized. Response time and error rate vary as function of stimulus orientation, reflecting the anatomical constraints of actual leg movements. Alternatively, this laterality judgment task can be solved by referring to a prototypical body schema, the "body structural description" (Corradi-Dell'Acqua et al. 2009), or visual transformation of the stimuli.

In addition to applying this task to lower limb amputees, we also tested a patient with a far more dramatic change of lower limb functional anatomy. This patient had undergone a Van Nes rotationplasty, a rare surgical intervention in which a diseased part of the femur bone and the knee is removed and the lower leg is re-attached to the thigh by a rotation through $180^{\circ}$ (Fig. 1; Van Nes 1950). The ankle now acts as a knee joint while the foot is facing backward. With this surgery the sciatic nerve is preserved. Further, patients do not experience phantom pain that is common in amputees (Grimer 2005). In order to walk with a prosthesis, the motor control system is forced to adapt to the changed anatomical situation. Bending and stretching of the ankle joint result in altered movement effects. Hence, these adaptations should also be reflected in the mental transformation of feet, if the patient refers to her own body structure.
Fig. 1 Rotationplasty. $40 \times 103 \mathrm{~mm}(600 \times 600 \mathrm{DPI})$

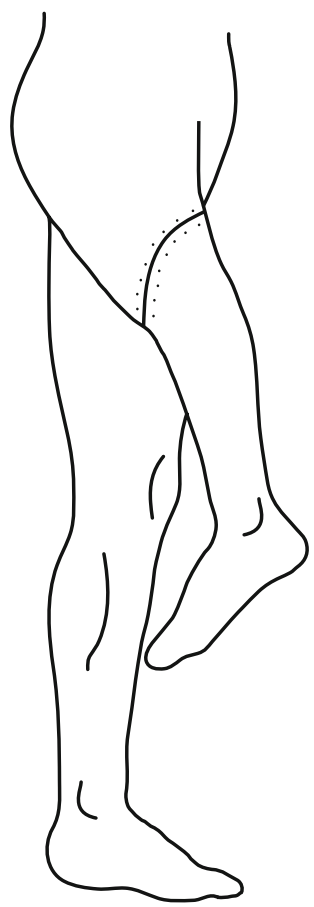

The goal of this study is to gain insight into the changes in body representation that take place due to lower limb surgery by means of a laterality judgment task that is assumed to require motor imagery (Bonda et al. 1995; Parsons et al. 1995). Determining the effects of absence of $a$ limb (amputation) opposed to a case of transformation of a limb (rotationplasty) on mental rotation will advance our understanding of body representation.

For the group of amputees, a selective impairment of mental rotation for the absent limb was hypothesised, as was found for upper limb amputees (Nico et al. 2004). Furthermore, studies in healthy people have suggested that mental rotation depends on the actual posture of the physical counterpart of one's own body (de Lange et al. 2006; Ionta et al. 2007). If mental rotation is sensitive to these transformations, this should be reflected in the performance data of the patient with a rotationplasty.

\section{Participants}

Eighteen participants (14 male, 4 female; mean age $62.8 \pm 9.7$ years) with a lower limb amputation (7 right, 11 left; level of amputation: 9 transtibial, 9 transfemoral) participated in the study (Table 1). The most frequent reasons for amputation were vascular disease (8), followed by trauma (7) and cancer (3). The mean time since amputation was $21.7 \pm 20.2$ years. All participants stated that they occasionally experience a phantom limb and are able to perform voluntary movements with their phantom. Additionally, one female participant (37 years) with a rotationplasty (right 
Table 1 Participant characteristics

\begin{tabular}{|c|c|c|c|c|c|c|c|c|c|c|c|c|}
\hline & \multirow[t]{2}{*}{ Sex } & \multirow{2}{*}{$\begin{array}{l}\text { Age } \\
\text { (year) }\end{array}$} & \multirow[t]{2}{*}{ Handedness } & \multicolumn{6}{|c|}{ Amputation } & \multicolumn{3}{|c|}{ Phantom limb } \\
\hline & & & & Side & Footedness & Level & $\begin{array}{l}\text { Time } \\
\text { (year) }\end{array}$ & Cause & $\begin{array}{l}\text { Prosthetic } \\
\text { use }\end{array}$ & General & $\begin{array}{l}\text { During } \\
\text { testing }\end{array}$ & Quality \\
\hline \multicolumn{13}{|c|}{ Amputees } \\
\hline 1 & M & 51 & $\mathrm{R}$ & $\mathrm{L}$ & $\mathrm{R}$ & Transtibial & 26 & Cancer & Yes & Yes & No & - \\
\hline 2 & M & 55 & $\mathrm{R}$ & $\mathrm{L}$ & $\mathrm{R}$ & Transfemoral & 4 & Vascular & Yes & Yes & No & - \\
\hline 3 & $\mathrm{~F}$ & 65 & $\mathrm{R}$ & $\mathrm{L}$ & $\mathrm{R}$ & Transfemoral & 46 & Trauma & Yes & Yes & No & - \\
\hline 4 & M & 71 & $\mathrm{R}$ & $\mathrm{L}$ & $\mathrm{R}$ & Transtibial & 15 & Vascular & Yes & Yes & No & - \\
\hline 5 & M & 62 & $\mathrm{R}$ & $\mathrm{L}$ & $\mathrm{R}$ & Transfemoral & 1 & Vascular & Yes & Yes & No & - \\
\hline 6 & M & 60 & $\mathrm{R}$ & $\mathrm{L}$ & $\mathrm{R}$ & Transfemoral & 41 & Trauma & Yes & Yes & No & - \\
\hline 7 & M & 49 & $\mathrm{R}$ & $\mathrm{L}$ & $\mathrm{R}$ & Transfemoral & 7 & Vascular & Yes & Yes & No & - \\
\hline 8 & M & 68 & $\mathrm{R}$ & $\mathrm{L}$ & $\mathrm{R}$ & Transtibial & 7 & Vascular & Yes & Yes & No & - \\
\hline 9 & M & 68 & $\mathrm{R}$ & $\mathrm{L}$ & $\mathrm{L}$ & Transfemoral & 3 & Vascular & Yes & Yes & No & - \\
\hline 10 & $\mathrm{~F}$ & 66 & $\mathrm{R}$ & $\mathrm{L}$ & $\mathrm{R}$ & Transfemoral & 9 & Trauma & Yes & Yes & No & - \\
\hline 11 & M & 49 & $\mathrm{R}$ & $\mathrm{L}$ & $\mathrm{R}$ & Transfemoral & 30 & Trauma & Yes & Yes & No & - \\
\hline 12 & $\mathrm{~F}$ & 57 & $\mathrm{R}$ & $\mathrm{R}$ & $\mathrm{R}$ & Transtibial & 5 & Cancer & Yes & Yes & No & - \\
\hline 13 & $\mathrm{~F}$ & 66 & $\mathrm{R}$ & $\mathrm{R}$ & $\mathrm{R}$ & Transtibial & 39 & Cancer & Yes & Yes & Yes & $\begin{array}{l}\text { Resting in } \\
\text { normal } \\
\text { orientation }\end{array}$ \\
\hline 14 & M & 78 & $\mathrm{R}$ & $\mathrm{R}$ & $\mathrm{R}$ & Transtibial & 58 & Trauma & Yes & Yes & No & \\
\hline 15 & M & 50 & $\mathrm{R}$ & $\mathrm{R}$ & $\mathrm{R}$ & Transtibial & 25 & Trauma & Yes & Yes & Occasionally & $\begin{array}{l}\text { Rotational } \\
\text { movements }\end{array}$ \\
\hline 16 & M & 76 & $\mathrm{R}$ & $\mathrm{R}$ & $\mathrm{R}$ & Transtibial & 63 & Trauma & Yes & Yes & No & - \\
\hline 17 & M & 60 & $\mathrm{R}$ & $\mathrm{R}$ & $\mathrm{R}$ & Transtibial & 3 & Vascular & Yes & Yes & No & - \\
\hline 18 & M & 79 & $\mathrm{R}$ & $\mathrm{R}$ & $\mathrm{R}$ & Transfemoral & 8 & Vascular & Yes & Yes & No & - \\
\hline \multicolumn{13}{|c|}{ Rotationplasty } \\
\hline 1 & $\mathrm{~F}$ & 37 & $\mathrm{~L}$ & $\mathrm{R}$ & $\mathrm{L}$ & Rotationplasty & 21 & Cancer & Yes & No & - & - \\
\hline
\end{tabular}

leg, time since surgery 21 years) took part in the study. All participants were prosthetic users, fitted with a mechanical prosthesis. Further, a group of 18 healthy control participants (13 male, 5 female; mean age $57.0 \pm 10.9$ years) was recruited. The protocol was approved by the local ethical committee. All participants gave their informed consent.

\section{Patient description: rotationplasty}

I.A. is a 37-year-old, left dominant, IT service assistant. She underwent a Van Ness rotationplasty of the right leg due to bone cancer at the age of 16 . Since then she became a highly active prosthetic user. The patient reports that in the first weeks after the surgery she experienced touches on the left of her affected limb as being touched on the right, and vice versa. Also the perception of the dorsal and ventral part was inverted. In this early stage the patient was dependent on visual feedback for the recalibration of this tactile afferent information. Nights were particularly stressful; then she experienced her affected leg hovering in the air while the bed's full weight was pressing down her affected leg. After reorganisation, she re-referenced tactile afferent information; thus, she was no longer dependent on visual feedback. However, till date, as was specified by the patient, she has to reckon the position of her big toe on the affected side.

\section{Stimuli}

Stimuli in the laterality recognition task were the original line drawings by Parsons (1987) and matching photos of prosthetic and natural feet (Fig. 2). The illustrations of left and right feet were mirror images. They depicted the dorsal and plantar view of feet in six different orientations (rotation angle, $60^{\circ}$ steps). This results in a total of 72 different stimuli. The zero orientation was defined as toes pointing up for the dorsal view and toes pointing down for the plantar view.

\section{Design and procedure}

The study was designed as an experimental, cross-sectional study with a control group. Participants were required to 
Fig. 2 Stimuli. Exemplary stimulus material of the right foot; the corresponding left foot stimuli were mirror images. Stimuli were presented in two views (dorsal, plantar) and six different orientations $\left(60^{\circ}\right.$ steps $)$. $168 \times 103 \mathrm{~mm}$ $(600 \times 600 \mathrm{DPI})$
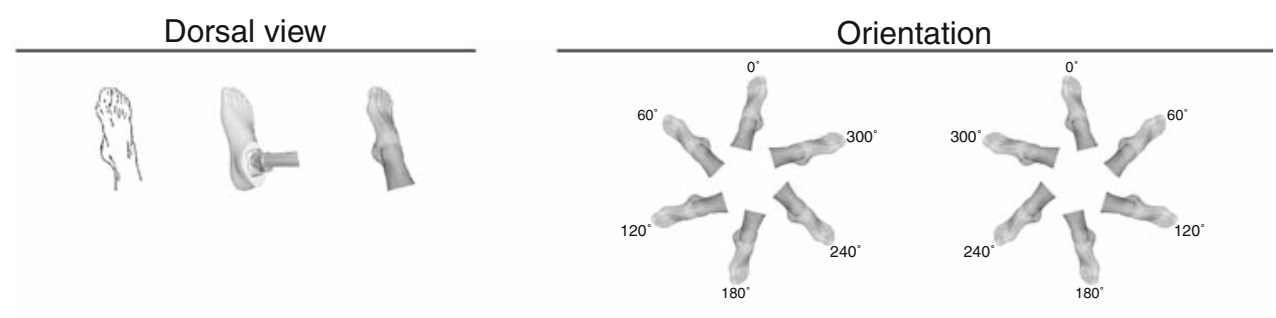

Plantar view

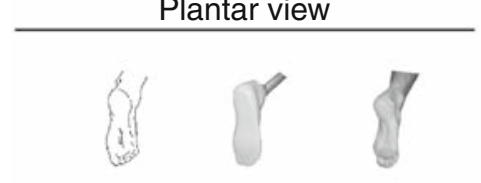

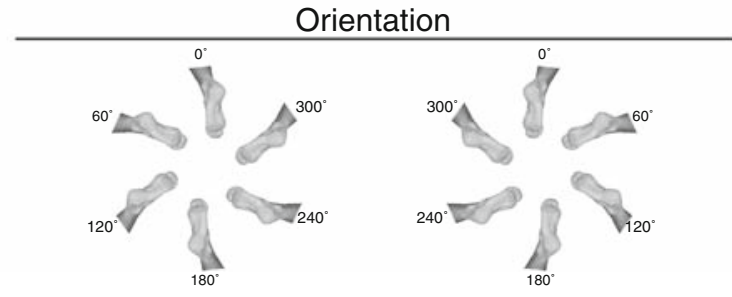

judge foot illustrations that were presented as single images on a monitor for their laterality. The participants sat comfortably in front of the computer, with their left and right index finger placed on the two response keys. They positioned their feet parallel and out of sight under the table. They were instructed not to move their feet during the experiment. Preceding each stimulus, a fixation cross appeared in the middle of the blank screen and remained visible for $1 \mathrm{~s}$. As it disappeared the stimulus was presented in the same location. The participants were instructed to respond as quickly and as correctly as possible by pressing the left or right key (corresponding to identification as left or right limb). If they did not respond within a time window of $5 \mathrm{~s}$, the test proceeded to the next trial automatically. The sequence of stimuli was randomized for each participant.

The participants completed a practice phase, in which each of the 72 different stimuli was presented to them once. The subsequent experimental phase consisted of 4 blocks with 144 trials each.

\section{Data analysis}

Analyses included response time for accurate response trials only. Trials with response times below $500 \mathrm{~ms}$ and above 3,500 ms were excluded from further analyses. To correct for skewness of the data distribution, response times were logarithmically transformed. The error rate was calculated and arcsine transformed.

Two separate three-way ANOVAs for repeated measures with stimulus laterality (left, right), view (plantar, dorsal) and orientation $\left(0^{\circ}, 60^{\circ}, 120^{\circ}, 180^{\circ}, 240^{\circ}\right.$ and $\left.300^{\circ}\right)$ as within-subject factors were performed on the response time and the error rate of the controls.
To evaluate the effect of lower limb amputation, two separate four-way ANOVAs with stimulus laterality (affected, contralateral), view (plantar, dorsal), orientation $\left(0^{\circ}, 60^{\circ}, 120^{\circ}, 180^{\circ}, 240^{\circ}, 300^{\circ}\right)$ as within-subject factors, and amputated limb (left, right) as between-subject factor were run on the response time and the error rate.

To determine the effect of the presence/absence of phantom sensations during the task two separate four-way ANOVAs were run on the response time and the error rate. The within-subject factors were stimulus laterality (affected, contralateral), view (plantar, dorsal), and orientation $\left(0^{\circ}, 60^{\circ}, 120^{\circ}, 180^{\circ}, 240^{\circ}, 300^{\circ}\right)$; and phantom (presence/absence of occasional phantom sensations during the task) was the between-subject factor.

Furthermore, to evaluate the effect of time since amputation, two separate analyses of covariance with stimulus laterality (affected, contralateral), view (plantar, dorsal), and orientation $\left(0^{\circ}, 60^{\circ}, 120^{\circ}, 180^{\circ}, 240^{\circ}, 300^{\circ}\right)$ as within-subject factors and time since amputation as covariate were performed.

To compare the controls with the amputees, two separate four-way ANOVAs were run on the response time and the error rate. The within-subject factors were stimulus laterality (left/affected, contralateral/right), view (plantar, dorsal), and orientation $\left(0^{\circ}, 60^{\circ}, 120^{\circ}, 180^{\circ}, 240^{\circ}, 300^{\circ}\right)$; and group (controls, amputees) was the between-subject factor.

To further analyse the effect of lower limb surgery, ratios between affected and contralateral limb were calculated from the logarithmic transformed response time (RT) data $\left[\ln \left(\mathrm{RT}_{\text {affected }} / \mathrm{RT}_{\text {contralateral }}\right)\right]$ for the amputees and the patient with rotationplasty, as well as for the left and right limb of healthy controls.

Contrasting amputees and rotationplasty, two-tailed one-sample $Z$ tests were used to determine if the observed 
logarithmically transformed ratios in response time are likely to come from different populations.

\section{Results}

Controls

The three-way ANOVA on the response time data indicated significant main effects of view $[F(1,17)=115.50$, $p<0.001]$, and orientation $[F(5,85)=137.38, p<0.001]$. Feet in the plantar view were considerably more difficult to recognize than feet in the dorsal view (Fig. 3). Controls were also faster when recognizing feet presented in a more natural orientation. Moreover, the interaction between view $\times$ orientation reached statistical significance $[F(5$, $85)=29.81, p<0.001]$, while the interaction between stimulus laterality $\times$ orientation was non-significant $[F(5$, $85)=1.21, p<0.309]$. Furthermore, there was a significant main effect for stimulus laterality $[F(1,17)=13.76$, $p=0.002]$, indicating faster mental rotation for right than left feet.

Analyses of the error rate (Fig. 4) confirmed these significant main effects [view $F(1,17)=14.15, p=0.002$; orientation $F(5,85)=7.63, p<0.001 ;$ stimulus laterality $F(1$, $17)=1.39, p=0.254]$. The interaction view $\times$ orientation $[F(5,85)=4.25, p=0.002]$, while the interaction of stimulus laterality $\times$ orientation remained non-significant $[F(5$, $85)=0.99, p<0.429]$.
Amputees

For the amputees, we found significant main effects on response time for view $[F(1,16)=202.92, p<0.001]$, and orientation $[F(5,80)=74.54, p<0.001]$ as well as a significant interaction between these two [view $\times$ orientation $F(5,80)=12.80, p<0.001]$, just as for healthy controls. Interestingly, no significant main effects were observed for either stimulus laterality $[F(1,16)=0.62, p=0.445]$ or amputated limb $[F(1,16)=0.01, p=0.927]$, nor the interaction between those two [stimulus laterality $\times$ amputated limb $F(1,16)=3.18, p=0.094]$. Only the interaction of stimulus laterality $\times$ orientation $[F(5,80)=2.41, p=0.044]$ reached some level of statistical significance. Remarkably, the presence/absence of a phantom limb during task execution did have a statistically significant effect on the laterally judgments [stimulus laterality $\times$ orientation $\times$ phantom $(F(5,80)=5.44, p<0.001)]$. Time since amputation, however, did not have a statistically significant effect on task performance [stimulus laterality $\times$ time since amputation $F(1,16)=2.93, p=0.106]$. Further, amputees who had lost their dominant limb did not perform any different from amputees who had lost their non-dominant limb [stimulus laterality $\times$ dominant/non-dominant limb loss $F(1,16)=$ $1.92, p=0.185]$.

Analysis of the error rate confirmed these findings [view $F(1,16)=39.78, p<0.001 ;$ orientation $F(5,80)=18.88$, $p<0.001$; view $\times$ orientation $F(5,80)=8.63, p<0.001$; stimulus laterality $F(1,16)=0.01, p=0.911 ;$ amputated
Fig. 3 Response times. Mean response times by group (controls $(n=18)$, amputees $(n=18)$ and rotationplasty $(n=1))$ for different views (dorsal, plantar) and stimulus laterality (affected/ left and contralateral/right) are shown. Error bars depict \pm 1SD. $159 \times 127 \mathrm{~mm}$ $(600 \times 600 \mathrm{DPI})$
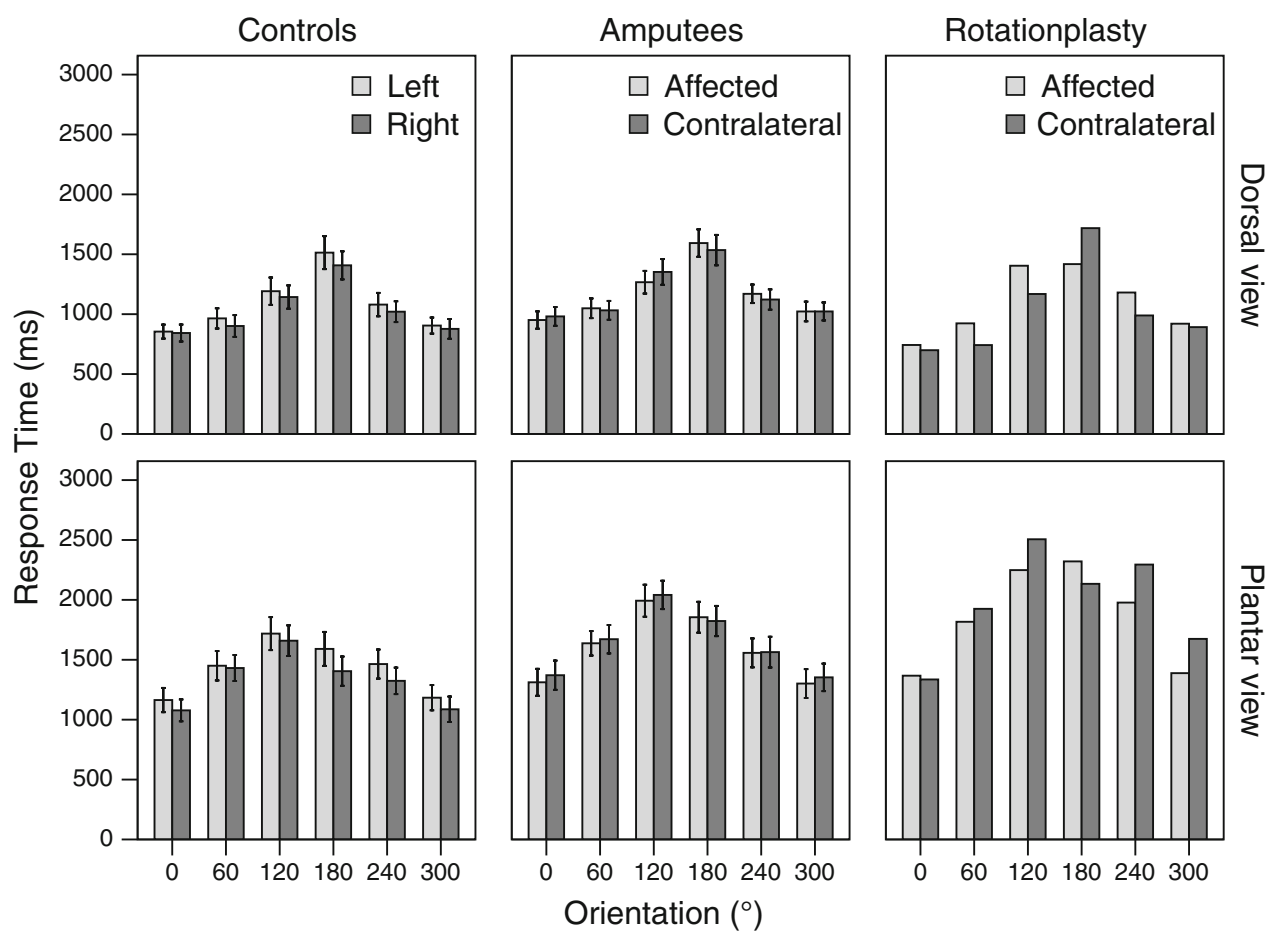
Fig. 4 Error rate. Mean error rate by group (controls $(n=18)$, amputees $(n=18)$ and rotationplasty $(n=1))$ for different stimulus laterality (affected/left and contralateral/right) are shown. Error bars depict \pm 1SD. $152 \times 116 \mathrm{~mm}$ $(600 \times 600 \mathrm{DPI})$ views (dorsal, plantar) and
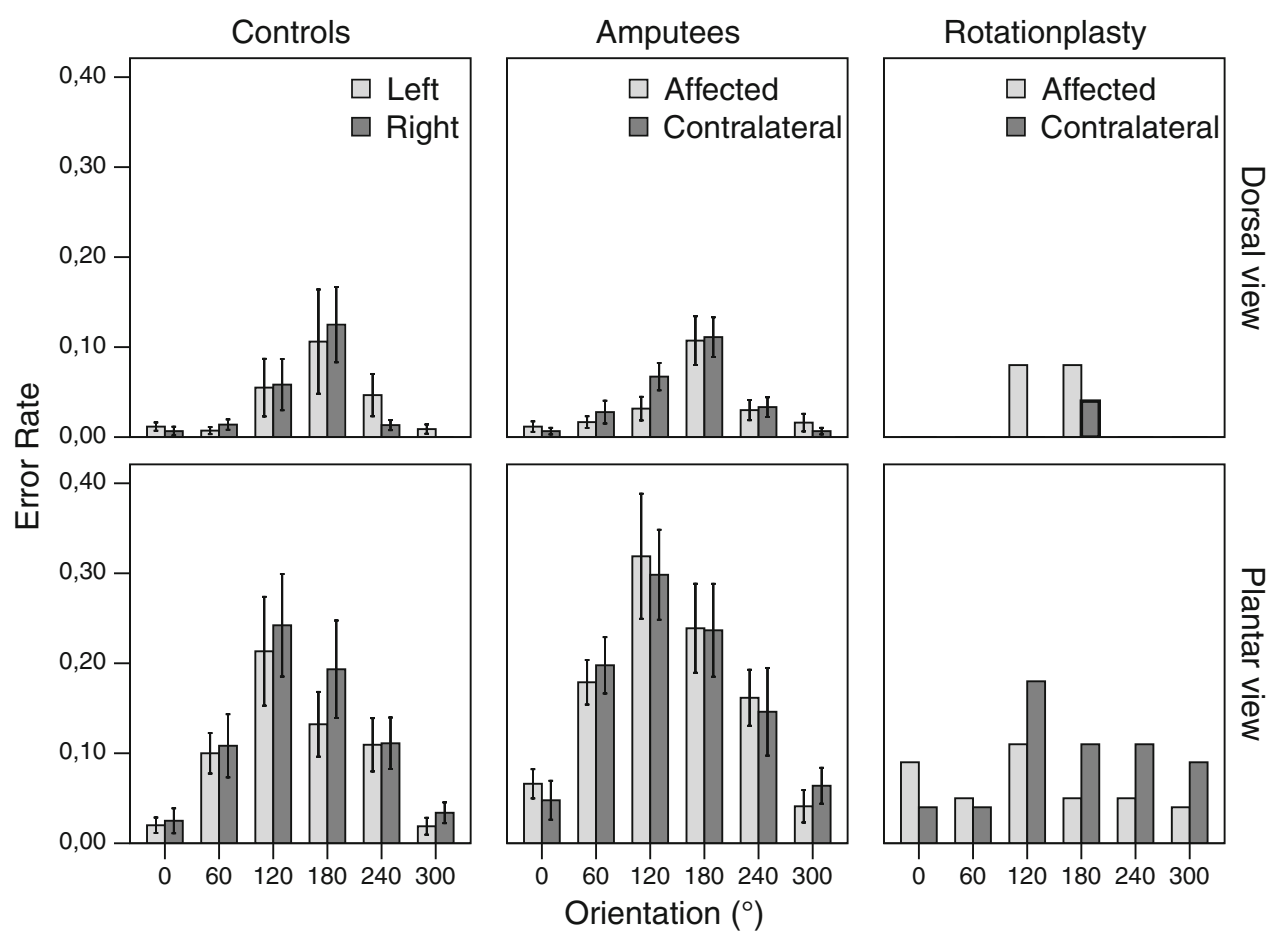

limb $F(1,16)=0.53, p=0.478 ;$ stimulus laterality $\times$ amputated limb $F(1,16)=2.18, \quad p=0.159 ;$ stimulus laterality $\times$ orientation $(F(5,80)=0.14, p=0.984]$.

Contrasting the group of amputees with the controls in two separate four-way ANOVAs, no significant main effect emerged for the factor group [response time: $F(1$, $34)=2.07, \quad p=0.159$; error rate: $F(1,34)=1.37$, $p=0.250]$. We found a significant main effect for the factor view [response time: $F(1,34)=323.12, p<0.001$; error rate: $F(1,34)=50.61, p<0.001]$. There was no significant interaction between view $\times$ group found for response time $[F(1,34)=2.70, \quad p=0.110]$, but only for error rate $[F(1,34)=4.18, p=0.049]$. Additionally, the orientation of stimuli had a significant effect on task performance [response time: $F(5,170)=201.38, p<0.001$; error rate: $F(5,170)=22.69, p<0.001]$, but there was no significant interaction between orientation $\times$ group [response time: $F(5,170)=0.57, p=0.726$; error rate: $F(5,170)=0.209$, $p=0.958]$. Moreover, analyses revealed a significant main effect for stimulus laterality [response time: $F(1,34)=4.86$, $p=0.034$; error rate: $F(1,34)=0.31, p=0.580]$. The interaction between stimulus laterality $\times$ group was significant only for response time $[F(1,34)=7.62, p=0.009]$ but not for error rate $[F(1,34)=0.22, p=0.640]$.

In a covariance analysis on all subjects (controls and amputees), we checked for a possible influence of motor preferences (handedness) on the results. This analysis showed no interaction for stimulus laterality $\times$ handedness $[F(1,35)=0.017, p=0.898]$, so we conclude that the laterality effect is not due to differences in motor preferences.

\section{Rotationplasty}

Visual inspection of the response time data of the patient with rotationplasty (Fig. 3) revealed the characteristic effects of orientation and view. Likewise, the error rate increased with the degree of orientations and the plantar view (Fig. 4), just as for all other participants. Despite the reverse foot position of the affected limb, there was no apparent interaction between orientation $\times$ stimulus laterality. Merely a slight trend towards faster and more accurate responses for the affected limb for the plantar view, but not the dorsal view, can be observed.

In Fig. 5, the ratios between affected and unaffected limb are shown for comparison between amputees and rotationplasty. As shown, the intervals of $2 \times$ SD encompass the mean values of the rotationplasty patient, indicating that these ratios fit within the normal range of amputees, as well as controls. Two-tailed one-sample $Z$ tests yielded no significant group differences on response times (dorsal: $p \geq 0.11$; plantar: $p \geq 0.29$ ).

Post hoc power analysis

The fact that we did not detect a main effect of stimulus laterality (affected, contralateral) for the amputee group could possibly be attributed to a lack of power. We performed a post hoc power analysis, assuming a significance level $\alpha=0.05$. Given our sample size of 18 amputees, we should be able to detect medium effects $(\Delta=0.25)$ of stimulus laterality with high power $(1-\beta=0.99)$, and small effects 


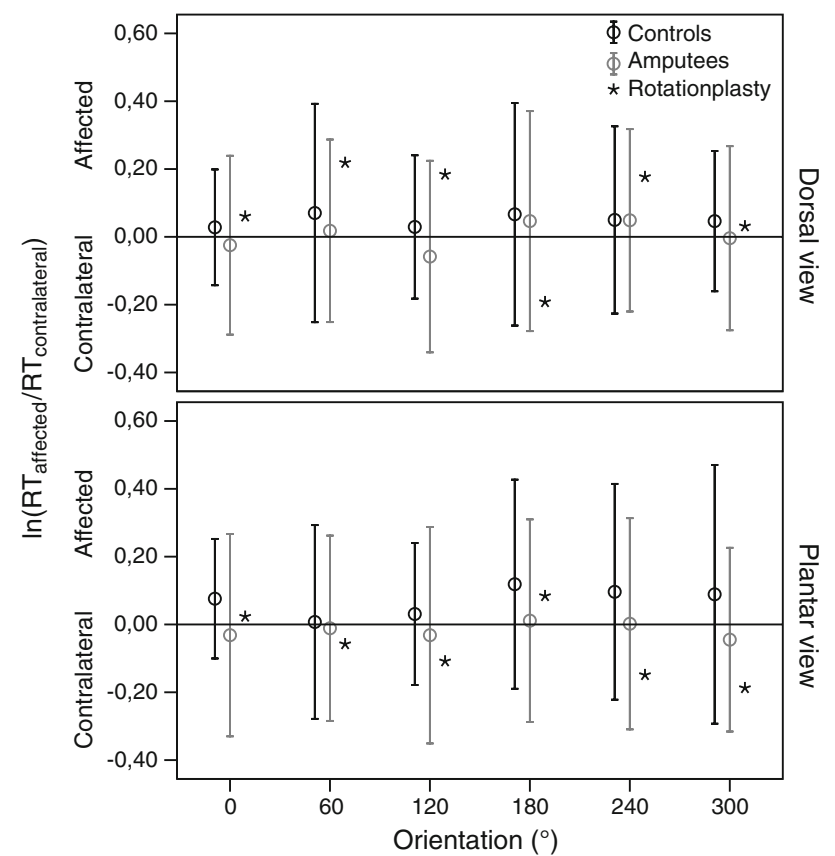

Fig. 5 Response time ratios. Mean ratios of response time $(R T)$ of affected/left and contralateral/right limb for different views (dorsal, plantar) and orientations. Error bars depict $\pm 2 \mathrm{SD}$ to indicate the significance level of the $Z$ test (see Data analysis). $117 \times 120 \mathrm{~mm}$ $(600 \times 600 \mathrm{DPI})$

$(\Delta=0.10)$ with moderate power $(1-\beta=0.55)$ (Cohen 1988). Thus, it is unlikely that we would have missed an existing medium main effect of stimulus laterality due to lack of power.

\section{Discussion}

In the present study, we measured chronometric changes in the mental rotation of feet as a consequence of lower limb amputation and rotationplasty. Our data confirm the typically strong effects of view, orientation and stimulus laterality found in healthy controls (de Lange et al. 2006; Ionta et al. 2007), suggesting mental rotation of the subject's own physical counterpart into the position of the stimulus. The effect of stimulus laterality, i.e. right feet were recognized faster than left feet, is most likely to be explained by foot dominance (Parsons 1987).

Our data on lower limb amputees indicate that these patients maintained their ability to perform mental rotations of feet representing the lost limb. Interestingly, no differences were observed in response time and error rate for judgments based on the affected versus contralateral limb. However, some differences in response were found between affected and contralateral limb, when accounting for the orientation of stimulus, but this interaction effect was not confirmed on the level of error rate. Furthermore, no differences in response were found between amputees who had lost their dominant or non-dominant limb. These findings are contrary to reports by Nico et al. (2004) for upper limb amputees; they showed that upper limb loss significantly impaired performance in the same task, notably if the dominant limb was amputated.

Remarkably, the presence/absence of phantom sensation during testing did have an effect on the laterally judgments in our amputee group.

Evidence for the robustness of internal action representation comes from chronic hemiplegics (Johnson 2000; Johnson et al. 2002). These patients preserved their ability to mentally simulate movements of the paralysed body part. Our results on a patient with rotationplasty illustrate that, even years after rotationplasty surgery, the patient retained the ability of unconstrained mental rotation. This is even more remarkable considering that the patient had adapted her motor behaviour. Rotationplasty patients, unlike amputees, receive sensory input from the periphery, i.e. their affected, but still present limb. As a consequence, we expected the patient to show a reversion in response time pattern for the rotated limb, if referring to her own body structure, since the post-surgical reorientation of her foot is different by $180^{\circ}$ from a prototypical one. However, a slightly better performance for the affected limb in the plantar view can be seen. This trend may suggest some effect of the inversion of the foot in the rotationplasty patient; however, the effect is only a slight trend which may as well fit into the normal range. Ionta et al. (2007) propose that the actual posture and the joined afferent information influence mental rotation of the same body part. However, the presented chronometric findings do not support this notion. The altered anatomical constraints are neither reflected in the response time nor in the error rate.

Berlucchi and Aglioti (1997) proposed that, despite the plasticity in the somatosensory cortex, the brain might be genetically predisposed to representing a prototypical human body. There is abundant evidence from neonatal research, as well as from phocomelic children for an innate origin of the body schema. In a landmark experiment, Meltzoff and Moore (1977) showed that neonates are capable of imitating orofacial and head movements of an adult model already days after delivery. This implies that they can equate their own and other people's body structure. Reports of phantom limb sensations in children with congenital limb deficiency provide further evidence for an innate body schema (Melzack et al. 1997). According to Melzack et al. (1997), the basic experience of the body is not a passive process that merely reflects sensory input from the body, but an interaction of genetic and sensory determinants. Evidence for multiple independent body representations comes from research by CorradiDell'Acqua et al. (2009). They could show that the brain is endowed with two representations of the body maintained 
by different neural substrates: (1) the body schema, which represents the orientation of one's own body in space, and (2) the body structural description, representing the location of body parts relative to a prototypical body.

Laterality emerges very early in life and when dealing with motor imagery, laterality effects provide reliable evidence that subjects are referring to a representation of their own body, as found here in the group of healthy controls. Accordingly, the disappearance of this functional marker represents a strong suggestion that the patients are not referring to a representation of their own body. Our findings suggest that the amputees and the patient with rotationplasty may be using a more abstract (prototypical) representation of the body, or more likely they may be operating mental visual transformations of the stimulus, thus not using motor imagery at all. It should be noted that mental rotation of a visual stimulus (rotating the picture of a foot, as opposed to imagining moving "my preferred foot") would entail exactly the disappearance of the laterality effect, while the effect of distance from a canonical orientation (i.e., a foot with the big toe pointing upward) would still be present. However, the elicitation of phantom sensations during the task in 2 of the 18 amputees suggests that at least these 2 patients were using motor imagery. This hypothesis should be tested in further studies, possibly making use of functional brain imaging during mental rotation tests in amputees.

Open Access This article is distributed under the terms of the Creative Commons Attribution Noncommercial License which permits any noncommercial use, distribution, and reproduction in any medium, provided the original author(s) and source are credited.

\section{References}

Berlucchi G, Aglioti S (1997) The body in the brain: neural bases of corporeal awareness. Trends Neurosci 20:560-564

Blakemore SJ, Wolpert DM, Frith CD (2002) Abnormalities in the awareness of action. Trends Cogn Sci 6:237-242

Bonda E, Petrides M, Frey S, Evans A (1995) Neural correlates of mental transformations of the body-in-space. Proc Natl Acad Sci USA 92:11180-11184

Buxbaum LJ, Coslett HB (2001) Specialized structural descriptions for human body parts: evidence from autotopagnosia. Cogn Neuropsychol 18:363-381

Cohen J (1988) Statistical power analysis for the behavioral sciences. Erlbaum, New York
Corradi-Dell' Acqua C, Tomasino B, Fink GR (2009) What is the position of an arm relative to the body? Neural correlates of body schema and body structural description. J Neurosci 29:4162-4171

de Lange FP, Hagoort P, Toni I (2005) Neural topography and content of movement representations. J Cogn Neurosci 17:97-112

de Lange FP, Helmich RCG, Toni I (2006) Body posture influences motor imagery: an fMRI study. Neuroimage 33:609-617

Gaser C, Schlaug G (2003) Brain structures differ between musicians and non-musicians. J Neurosci 23:9240-9245

Grimer RJ (2005) Surgical options for children with osteosarcoma. Lancet Oncol 6:85-92

Head H, Holmes G (1911) Sensory disturbances from cerebral lesions. Brain 34:102-254

Ionta S, Fourkas AD, Fiorio M, Aglioti SM (2007) The influence of hands posture on mental rotation of hands and feet. Exp Brain Res 183:1-7

Johnson SH (2000) Imagining the impossible: intact motor representations in hemiplegics. Neuroreport 11:729-732

Johnson SH, Sprehn G, Saykin AJ (2002) Intact motor imagery in chronic upper limb hemiplegics: evidence for activity-independent action representations. J Cogn Neurosci 14:841-852

Knecht S, Henningsen H, Elbert T, Flor H, Höhling C, Pantev C, Taub E (1996) Reorganizational and perceptional changes after amputation. Brain 119:1213-1219

Meltzoff AN, Moore MK (1977) Imitation of facial and manual gestures by human neonates. Science 198:75-78

Melzack R (1992) Phantom limbs. Sci Am 266:120-126

Melzack R, Israel R, Lacroix R, Schultz G (1997) Phantom limbs in people with congenital limb deficiency or amputation in early childhood. Brain 120:1603-1620

Nico D, Daprati E, Rigal F, Parsons L, Sirigu A (2004) Left and right hand recognition in upper limb amputees. Brain 127:120-132

Parsons LM (1987) Imagined spatial transformations of one's hands and feet. Cognit Psychol 19:178-241

Parsons LM (1994) Temporal and kinematic properties of motor behavior reflected in mentally simulated action. J Exp Psychol Hum Percept Perform 20:709-730

Parsons LM, Fox PT, Downs JH, Glass T, Hirsch TB, Martin CC, Jerabek PA, Lancaster JL (1995) Use of implicit motor imagery for visual shape discrimination as revealed by PET. Nature 375:54-58

Pick A (1922) Störungen der Orientierung am eigenen Körper. Psychol Forsch 2:303-318

Ramachandran VS, Rogers-Ramachandran D (2000) Phantom limbs and neural plasticity. Arch Neurol 57:317-320

Schwoebel J, Coslett HB (2005) Evidence for multiple, distinct representations of the human body. J Cogn Neurosci 17:543-553

Schwoebel J, Friedman R, Duda N, Coslett HB (2001) Pain and the body schema: evidence for peripheral effects on mental representations of movement. Brain 124:2098-2104

Sirigu A, Grafman J, Bressler K (1991) Multiple representations contribute to body knowledge processing: evidence from a case of autotopagnosia. Brain 114:629-642

Van Nes CP (1950) Rotation-plasty for congenital defects of the femur. Making use of the ankle of the shortened limb to control the knee joint of a prosthesis. J. Bone Jt Surg 32:12-16 